\title{
Engineering valley quantum interference in anisotropic van der Waals heterostructures
}

\author{
Muralidhar Nalabothula $\odot,{ }^{1}$ Pankaj K. Jha, ${ }^{2}$ Tony Low ${ }^{3}$ and Anshuman Kumar $\odot^{1, *}$ \\ ${ }^{1}$ Physics Department, Indian Institute of Technology Bombay, Mumbai 400076, India \\ ${ }^{2}$ Thomas J. Watson Laboratories of Applied Physics, California Institute of Technology, Pasadena, California 91125, USA \\ ${ }^{3}$ Department of Electrical and Computer Engineering, University of Minnesota, Minneapolis, Minnesota 55455, USA
}

(Received 9 October 2019; revised 12 June 2020; accepted 15 June 2020; published 15 July 2020)

\begin{abstract}
In this paper, we present a novel route to manipulate the spontaneous valley coherence in two-dimensional valleytronic materials interfaced with other layered materials hosting anisotropic polaritonic modes. We propose two implementations - one, using anisotropic plasmons in phosphorene and another with hyperbolic phonon polaritons in $\alpha-\mathrm{MoO}_{3}$. In particular, we show the electrostatic tunability of the spontaneous valley coherence achieving robust valley coherence values in the near-infrared wavelengths at room temperature. The tunability of this valley coherence shown in these heterostructures would enable the realization of active valleytronic quantum circuitry.
\end{abstract}

DOI: 10.1103/PhysRevB.102.045416

An anisotropic material is one for which the magnitude of the optical conductivity tensor components is not the same along the crystal directions. The artificial realization of this anisotropy is key to the development of the emerging field of metasurfaces [1] and metamaterials [2,3] since they allow us to tailor the emission characteristics and wave propagation in ways completely different from the usual isotropic systems. Such materials are further classified into elliptical and hyperbolic systems where the nomenclature arises from the topology of the isofrequency surfaces [4]. Hyperbolic systems, in particular, allow access to large wave vectors via the so-called high- $k$ modes which enable several applications, such as strong spontaneous emission enhancement [5-8], subwavelength imaging $[9,10]$, thermal radiation enhancement [11-13], and biosensing [14]. The artificial realization of these anisotropic systems suffers from several challenges, the most important being the limitation on the maximum allowable wave vector which is restricted by the size of the metamaterial unit cell that can be achieved by nanofabrication $[15,16]$. Second, such metamaterials are usually passive elements, which cannot be modified postfabrication.

There has been a recent emergence of naturally occurring anisotropic materials $[17,18]$ where the anisotropy of the crystal unit cell leads to an anisotropic optical conductivity. This class of natural materials spans a broad spectrum of optoelectronic properties, and the anisotropy of the optical response ranges from ultraviolet to midinfrared wavelengths $[19,20]$. In this context, there has been a recent discovery of anisotropic optical response in monolayers of group-V compounds, in particular, phosphorene [21,22] and in transition-metal oxides, such as molybdenum trioxide [23]. The optical conductivity of the former is electronic in origin and shows elliptical nature in the visible to the near-infrared range, which is tunable via electrostatic doping. The lat-

*anshuman.kumar@iitb.ac.in ter, namely, molybdenum oxide shows hyperbolic response which originates from phonon polaritons in the midinfrared range.

In this paper, we present an unconventional application of this natural optical anisotropy in these van der Waals crystals to the field of quantum optics. Coherent superposition of states is a fundamental feature which distinguishes quantum mechanics from its classical counterpart. As such, quantum coherence is important from a fundamental physics perspective. This property is also critical for many of the emerging applications, such as quantum computation and communication. For practical applications, quantum coherence in solidstate systems is of paramount interest. Of particular interest recently are excitons in two-dimensional gapped Dirac systems, such as transition-metal dichalcogenides (TMDCs) [24] and biased bilayer graphene (BLG) [25]. The electronic band structure of such systems consists of two inequivalent yet degenerate valleys $\left(K\right.$ and $\left.K^{\prime}\right)$ in $k$ space for which the optical selection rule is sensitive to the helicity $\left(\sigma_{ \pm}\right)$of the exciting photon. This selection rule is inherited by excitons associated with these two valleys when Coulomb interaction is taken into account [26,27]. There have been several proposals to use this "valley degree of freedom" for the development of optoelectronic devices analogous to those in the field of spintronics [28].

In order to harness this valley degree of freedom, it is imperative to be able to control the coherence between excitons in the two valleys actively. Such a coherence has been demonstrated in the stimulated regime utilizing an external source, such as a laser [29] and has been theoretically proposed in the spontaneous regime, using anisotropic metasurfaces [30]. Here, we theoretically demonstrate how this spontaneous valley coherence can be achieved and manipulated by creating a heterostructure of the valley material interfaced with another anisotropic polaritonic (such as plasmonic or phononic) material. Unlike previous proposals, our proposed route to spontaneous valley coherence does not require spatial 


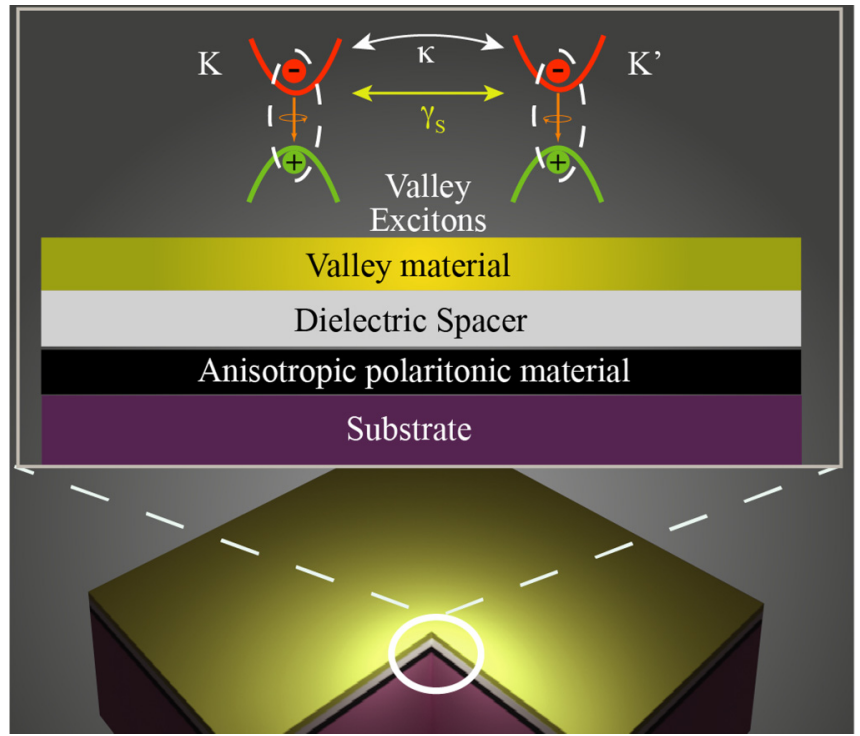

FIG. 1. Schematic of the heterostructure on a substrate. The heterostructure is designed such that an anisotropic vacuum of the electromagnetic field is created due to the $2 \mathrm{D}$ material, and radiation is focused back. The excitons in the valleys are modeled as in-plane circular dipoles.

patterning into nanostructures and allows for electrostatic tunability of the coherence.

Let us consider a valleytronic material sitting above another anisotropic polaritonic material, as shown in Fig. 1. The latter can be another naturally occurring two-dimensional (2D) material, which we will expound upon later. If we consider an exciton created in one valley $(K)$, then, in free space, this exciton decays radiatively by emitting a photon. This photon cannot excite an exciton in the orthogonal valley $\left(K^{\prime}\right)$ due to optical selection rule. However, in the presence of a neighboring anisotropic layered material which induces an in-plane anisotropic vacuum near the valleytronic material, the coupling between the two valleys are allowed through the quantum interference effect [31]. Thus, emission from one valley $(K)$ can excite an exciton in the orthogonal valley $\left(K^{\prime}\right)$. This effect is explained in Refs. [30,32,33] by employing a three-level $V$ scheme comprising two levels representing the $K$ - and $K^{\prime}$-valley excitons and one ground-state level. The quantum master equation of the interacting valley excitons in a weak-coupling regime under the rotating-wave approximation, second-order Born, and Markov approximations to the interaction Hamiltonian is given by [34-36]

$$
\begin{aligned}
\dot{\rho}= & \sum_{n, m} \Gamma_{n m}\left(\rho_{m n}|g\rangle\langle g|-\frac{1}{2}\{|n\rangle\langle m|, \rho\}\right) \\
& +R \sum_{n}\left[\left(\rho_{g g}|n\rangle\left\langle n\left|+\rho_{n n}\right| g\right\rangle\langle g|\right)-\frac{1}{2}\{|n\rangle\langle n|, \rho\}\right] \\
& +\gamma_{s} \sum_{n \neq m}\left[\rho_{n n}|m\rangle\langle m|-\frac{1}{2}\{|n\rangle\langle n|, \rho\}\right] \\
& -\gamma_{\text {dep }} \sum_{n \neq m}\left[\rho_{m n}|m\rangle\langle n|\right]+i \sum_{m, n} \delta_{n m}[|n\rangle\langle m|, \rho],
\end{aligned}
$$

where $\quad \Gamma_{m n}=\frac{2 \omega_{n}^{2}}{\hbar \epsilon_{0} c^{2}}\left[\mathbf{d}_{m}^{\dagger} \cdot \operatorname{Im}\left\{\mathbf{G}\left(\vec{r}_{0}, \vec{r}_{0} ; \omega_{n}\right)\right\} \cdot \mathbf{d}_{n}\right], \delta_{m n}=$ $\frac{1}{\hbar \epsilon_{0} \pi} \mathbb{P} \int_{0}^{\infty} d \omega \frac{\omega^{2}}{c^{2}} \frac{\left[\mathbf{d}_{m}^{\dagger} \cdot \operatorname{Im}\left\{\mathbf{G}\left(\vec{r}_{0}, \vec{r}_{0} ; \omega\right)\right\} \cdot \mathbf{d}_{n}\right]}{\omega-\omega_{n}}$, and $n, m \in\left\{K, K^{\prime}\right\}$ denote the valley indices of the exciton at position $\vec{r}_{0} . \Gamma_{n n}$ is the exciton decay rate, $\Gamma_{n \neq m}$ is the radiative coupling between two valleys, $\delta_{n n}$ is the Lamb shift, and $\delta_{n \neq m}$ is the dipole-dipole shifts. Here, $\omega_{n}$ is the exciton emission frequency, $\mathbf{d}_{n}$ is the transition dipole moment, $\mathbf{G}\left(\vec{r}_{0}, \vec{r}_{0}, \omega\right)$ is the total Green's tensor which is the sum of the vacuum and scattered Green's tensor, $R$ is the incoherent bidirectional pump rate, $\gamma_{s}$ is the intervalley scattering rate, and $\gamma_{\text {dep }}$ accounts for the pure dephasing rate.

Since in most valleytronic materials, the excitonic dipole at the two valleys is circularly polarized, the spontaneous coupling rate between the two valleys can be simplified as $\kappa=$ $\Gamma_{\mathrm{K}, \mathrm{K}^{\prime}}=\Gamma_{\mathrm{K}^{\prime}, \mathrm{K}}=\left(\gamma_{x}-\gamma_{y}\right) / 2$, where $\gamma_{i}$ is the spontaneous emission rate for an in-plane dipole sitting near the anisotropic material and oriented in the $i$ th direction. It is, thus, straightforward to see that any photonic environment which creates an anisotropy in the spontaneous emission rate in the $x$ and $y$ directions will result in the coupling between the two valleys, thus, leading to a finite generation of spontaneous valley coherence. Experimentally, the valley coherence is measured by a metric called the degree of linear polarization (DoLP) and using the valley-specific Jaynes-Cummings model given in Ref. [34], the DoLP can be written as

$$
\operatorname{DoLP}=\frac{I_{H}-I_{V}}{I_{H}+I_{V}}=\frac{\varrho_{K K^{\prime}}+\varrho_{K^{\prime} K}}{\varrho_{K K}+\varrho_{K^{\prime} K^{\prime}}},
$$

where $I_{H}$ and $I_{V}$ are the intensities of the horizontal and vertical polarized emissions, respectively, and $\varrho$ is the density matrix. The steady-state DoLP in the absence of pumping is an ill-defined quantity as the populations in $K\left(K^{\prime}\right)$ and valley coherence converge to zero. Therefore, to measure the spontaneous coherence generated, we assume a weak incoherent bidirectional pump. The exciton population dynamics are governed by the rate equations which are obtained from Eq. (1) and the Supplemental Material [37]. Using these rate equations, the steady-state DoLP can be written as

$$
\operatorname{DoLP}(t \rightarrow \infty)=-\frac{Q}{\left(1+\frac{R+\gamma_{s}+\gamma_{\mathrm{dep}}}{\gamma}\right)},
$$

where $\gamma=\Gamma_{K K}=\Gamma_{K^{\prime} K^{\prime}}=\left(\gamma_{x}+\gamma_{y}\right) / 2$. We define quantum interference strength as $Q=\frac{\kappa}{\gamma}=\frac{\gamma_{x}-\gamma_{y}}{\gamma_{x}+\gamma_{y}}$. Figure 2 shows the temporal evolution of the DoLP upon optical pumping. With an isotropic 2D material, the DoLP is zero (shown by the blue curve), whereas the presence of an anisotropic 2D material induces a time evolution of the DoLP which approaches a steady state $(\sim-Q)$, given by Eq. (3). The Fig. 2 inset shows the dependence of steady-state coherence in the presence of the intervalley decoherence rate which is equal to the sum of $\gamma_{s}$ and $\gamma_{\text {dep. Intervalley scattering is attributed to }}$ the electron-hole exchange interaction and phonon-assisted processes [38-41] where as the pure dephasing rate occurs due to disturbance in the phase relation between the two valleys which is attributed to the long-range part of the electronhole interaction that generates a momentum-dependent effective magnetic field, around which the valley pseudospin of 


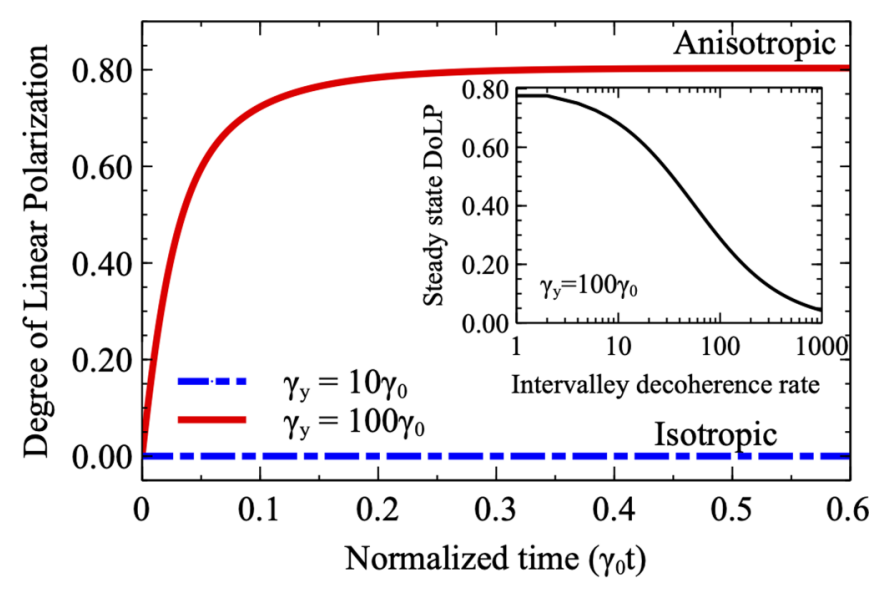

FIG. 2. Temporal evolution of the DoLP for different values of $\gamma_{y}$. The inset figure shows the dependence of the steady-state DoLP with the intervalley decoherence rate which is equal to the sum of $\gamma_{s}$ and $\gamma_{\text {dep }}$. For the entire figure, $\gamma_{x}=10 \gamma_{0}$, and the pumping rate is taken as $\left(R=\gamma_{0}\right)$. Initial conditions are taken as $\varrho_{K K^{\prime}}(0)=0$, $\varrho_{K K}(0)=0, \varrho_{K^{\prime} K^{\prime}}(0)=0, \varrho_{00}(0)=1$.

excitons with different center-of-mass momentum will precess with different frequencies [42-44].

As a demonstration of the valley coherence tunability, we first explore the parameter space of arbitrary optical conductivity tensor components of the anisotropic polaritonic 2D material. To do this, we calculate the spontaneous emission rates $-\gamma_{x}$ and $\gamma_{y}$ of an in-plane dipole, corresponding to an exciton situated at a distance $d$ above a general anisotropic 2D surface $[45,46]$. This rate is proportional to the imaginary part of the scattered component of the dyadic Green's function (in the presence of the anisotropic 2D surface), which is obtained using standard methods [47-49]. Figures 3(a)-3(d) show the

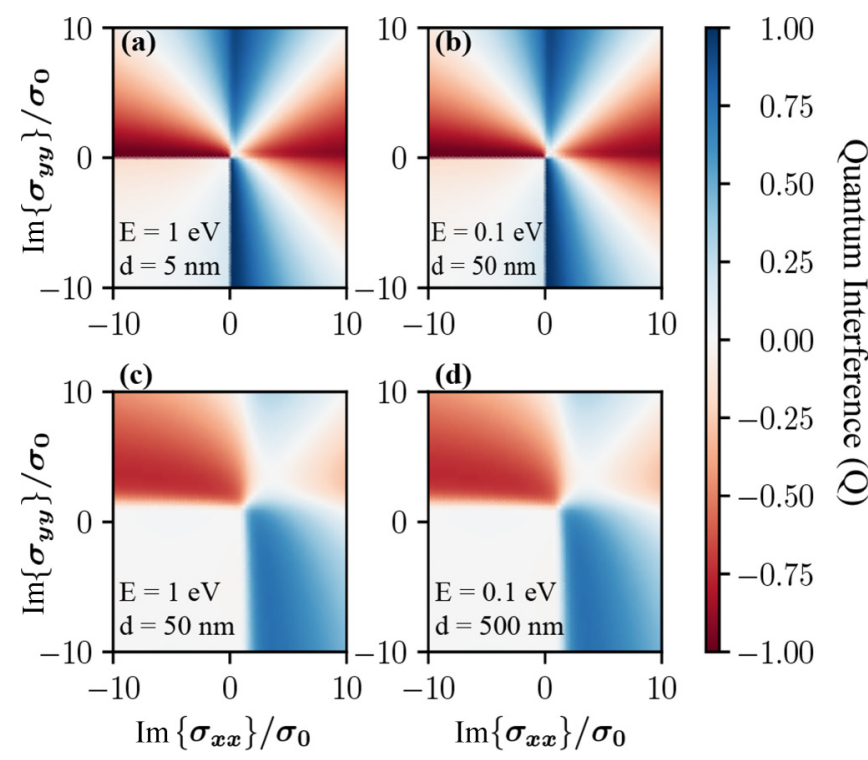

FIG. 3. (a)-(d) $Q$ maps. Dependence of quantum interference $(Q)$ on the diagonal conductivities for two dipole distances and exciton frequencies in low loss limit when the dipole is standing above the $2 \mathrm{D}$ anisotropic material. $\sigma_{0}=\frac{e^{2}}{\hbar}$ and $\operatorname{Re}[\sigma]=\frac{1}{1000} \operatorname{Im}[\sigma]$ for all the plots. dependence of $Q$ on the diagonal elements of the optical conductivity tensor for two distances and two exciton frequencies. Such diagrams can be constructed for other frequencies depending on the excitonic resonance for the valleytronic material in question. The four quadrants represent two hyperbolic regimes (second and fourth), that is, $\operatorname{Im}\left\{\sigma_{x x}\right\} \operatorname{Im}\left\{\sigma_{y y}\right\}<0$ and two elliptical regimes where $\operatorname{Im}\left\{\sigma_{x x}\right\} \operatorname{Im}\left\{\sigma_{y y}\right\}>0$ [50]. It should be noted that the first quadrant supports elliptical plasmons, whereas the third quadrant does not support any surface mode. It is clear from Figs. 3(a)-3(d) that the hyperbolic regime results in the largest values of the valley coherence. This can be intuitively understood based on the fact that a hyperbolic 2D system is metallic in one direction and insulating in the other. In the lossless limit, the metallic direction can support the strongly confined TM plasmon mode whereas the insulating direction supports only the weakly confined TE plasmon $[51,52]$. The large contrast between the respective mode volumes results in a strong anisotropy in the intravalley spontaneous emission rates in the two directions, leading to maximum valley coherence reaching close to the theoretical upper limit in the hyperbolic regions.

In the elliptical metallic region (first quadrant), we also observe the enhanced valley coherence. These regions correspond to an elliptical plasmon mode which can also produce an anisotropic spontaneous emission rate in the two directions. The contours where the valley coherence maximum occurs can again be tuned by changing the distance of the valleytronic material and the anisotropic surface. These $Q$ maps can be used to optimize the geometry of the heterostructure and enable the selection of the appropriate optical conductivity tensor of the anisotropic 2D material to achieve the desired valley coherence.

To interpret the features in the $Q$ maps of Figs. 3(a)-3(d) and find the optimal distance for the given conductivity and frequency, we employ the dispersion relation of the heterostructure. For an anisotropic 2D material, the dispersion relation of the propagating polariton when $k_{z} \gg k_{0}$ is given by [53]

$$
\sigma_{x x} \cos ^{2} \theta+\sigma_{y y} \sin ^{2} \theta=\frac{2 i \varepsilon_{0} \omega}{q} .
$$

Here, $\theta=\tan ^{-1} \frac{k_{y}}{k_{x}}$ is the propagation angle, $q=\sqrt{k_{x}^{2}+k_{y}^{2}}$ is the in-plane wave vector, $\sigma_{x x}$ and $\sigma_{y y}$ are optical conductivity tensors in the $x$ and $y$ directions, respectively, and $\omega$ is the frequency of the dipole. Since the dipole distance $d$ should approximately match the inverse in-plane wave-vector $1 / q$ to couple efficiently to the corresponding polariton [54], Eq. (4) becomes $\sigma_{x x} \cos ^{2} \theta+\sigma_{y y} \sin ^{2} \theta \approx 2 i \varepsilon_{0} \omega d$. In the lossless limit, we can assume $\sigma_{x x} \approx i \sigma_{x x}^{\prime \prime}$ and $\sigma_{y y} \approx i \sigma_{y y}^{\prime \prime}$ where $\sigma_{y y}^{\prime \prime}$ and $\sigma_{x x}^{\prime \prime}$ are real. The propagation angle is given by

$$
\theta= \pm \sin ^{-1} \sqrt{\frac{2 \varepsilon_{0} \omega d-\sigma_{x x}^{\prime \prime}}{\left(\sigma_{y y}^{\prime \prime}-\sigma_{x x}^{\prime \prime}\right)}} .
$$

In a heterostructure, the distance between the valleytronic material and the anisotropic 2D material can be varied through the use of a spacer layer. This distance has to be optimized for a given frequency and optical conductivity (which is also frequency dependent, in general) to efficiently excite the 

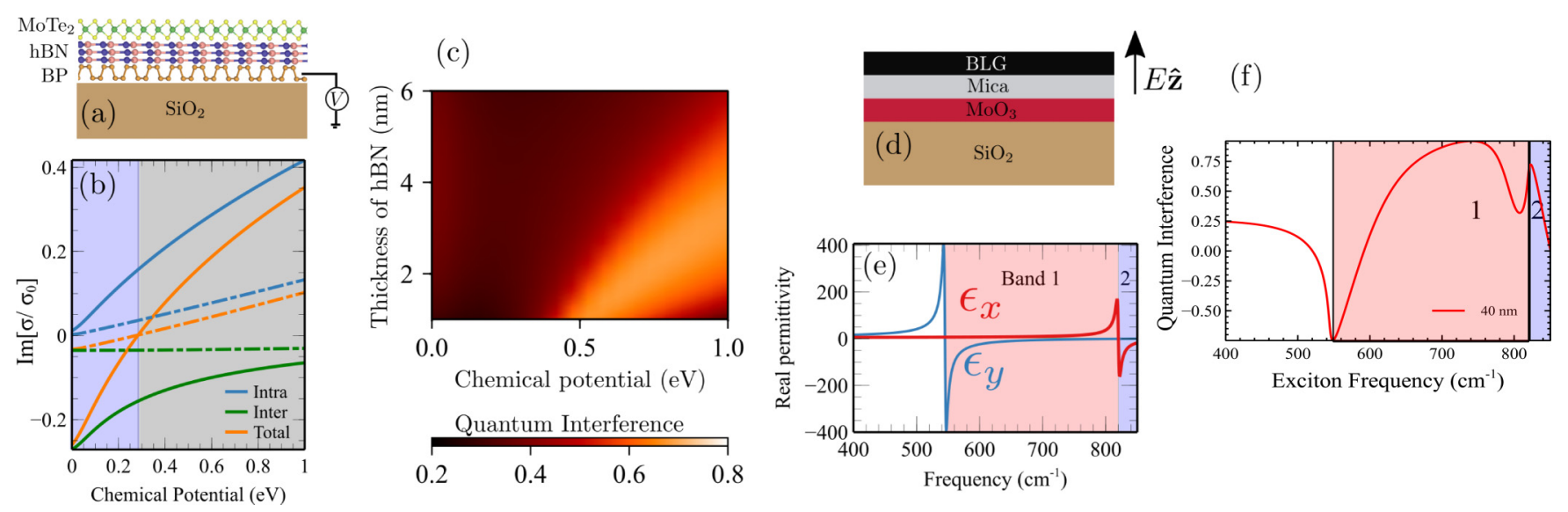

FIG. 4. (a)-(c) are for the TMDC/hexagonal boron nitride (hBN)/phosphorene heterostructure: (a) Schematic of the heterostructure. (b) Imaginary parts of conductivities of phosphorene (solid and dotted lines are for armchair and zigzag directions, respectively) vs chemical potential $(\mu)$ at $\mathrm{MoTe}_{2}$ exciton frequency $1.1 \mathrm{eV}$. The blue and gray shaded regions represent the elliptical insulator regime and elliptical metallic regime, respectively. (c) Color plot of quantum interference (Q) with hBN thickness and chemical potential ( $\mu$ ). The Fermi level is defined, and $E_{f}=E_{c}(\Gamma)+\mu$. The bright region represents the high valley coherence region. (d)-(f) are for the $\mathrm{BLG} / \mathrm{mica} / \alpha-\mathrm{MoO} 3$ heterostructure: (d) Schematic of the heterostructure. (e) Dielectric function of $\alpha-\mathrm{MoO}_{3}$. The white region represents the elliptical insulating regime. The orange and blue regions represent two hyperbolic bands. (f) Quantum interference (Q) as a function of the BLG exciton frequency in the heterostructure. The thickness of mica is equal to twice the thickness of $\mathrm{MoO}_{3}$.

polariton. To obtain higher coherence values, the polariton must be narrowly confined along one direction, i.e., $\theta \approx$ 0 or $90^{\circ}$, resulting in an anisotropic electric-field enhancement. From Eq. (5), the optimal distances for high coherences are given by $d=\sigma_{x x}^{\prime \prime} /\left(2 \varepsilon_{0} \omega\right)$ or $\sigma_{y y}^{\prime \prime} /\left(2 \varepsilon_{0} \omega\right)$. As shown in Fig. 3, an overall shift of the $Q$ maps is seen as a function of the distance $d$ of the dipole from the metasurface. This is because, in order to couple to a surface mode, the in-plane wave-vector $q$ must be larger than the gapped region of the isofrequency surface. This means that there is an optimal range of the conductivity tensor for a given distance $d$ where the isofrequency surface of the elliptical or the hyperbolic plasmon intersects [54], explaining the distance dependence. If the frequency of the exciton is varied, we obtain a qualitatively similar trend in the $Q$ maps as shown in Fig. 3 where we have compared the exciton frequency of 1 and $0.1 \mathrm{eV}$. This is because the minimum conductivity condition is determined only by the product $k_{0} d$. When the exciton strongly couples with the polariton, the decay rates are greatly enhanced. If the lifetime of the exciton is shorter than the lifetime of the polariton, we enter into the strong-coupling regime. The distance of the emitter is kept sufficiently long so that we are in the weak light-matter-coupling limit.

In order to realize the anisotropic quantum vacuum, we propose two layered materials which support anisotropic polaritons. In the following, we focus on plasmon and phonon polaritons. In both cases, we propose the tunability of spontaneous valley coherence by active and passive routes, respectively. As the first example heterostructure for demonstrating spontaneous valley coherence, we consider a naturally inplane anisotropic material, phosphorene [55,56] placed near a TMDC which supports valley excitons. Figure 4(a) illustrates this system with phosphorene sandwiched between a $\mathrm{SiO}_{2}$ substrate and a finite thickness h-BN dielectric [57]. Monolayer $\mathrm{MoTe}_{2}$ [58,59] is placed on top of the h-BN. The geometrical anisotropy of the unit cell of phosphorene translates to an anisotropic optical conductivity $[21,60]$. The optical conductivity of phosphorene in the armchair and zigzag directions as a function of chemical potential $\mu$ is shown in Fig. 4(b). The blue region is the elliptical insulator regime where $\operatorname{Im}\left\{\sigma_{x x}\right\}, \operatorname{Im}\left\{\sigma_{y y}\right\}<0$, and the gray region is the elliptical metallic regime where $\operatorname{Im}\left\{\sigma_{x x}\right\}, \operatorname{Im}\left\{\sigma_{y y}\right\}>0$. This chemical potential modification can be controlled via electrostatic gating [61-63] or chemical routes [64,65]. Doping provides an easy route to realize an actively tunable natural anisotropic environment for the monolayer TMDC, which enables one to modulate the valley coherence. For very small thicknesses of $\mathrm{hBN}$, one needs to consider nonlocal corrections to the optical conductivity of phosphorene. Recent calculations [66] have shown, however, that at our frequency of interest, the spontaneous emission rate is insensitive to nonlocal effects for dipole distances greater than $\sim 2 \mathrm{~nm}$.

Figure 4(c) illustrates the color plot of the $Q$ depending on h-BN thickness and chemical potential $(\mu)$. We have considered chemical potentials below $1 \mathrm{eV}-\mathrm{a}$ range which has been shown to be achievable with electrostatic doping techniques [61-63,67]. Figure 4(c) further shows that, as the thickness of $\mathrm{hBN}$ is increased, the interaction of the exciton with the surface plasmon modes decreases, yielding low coherence values. The bright region (high valley coherence) starts when $\mu \sim 0.3 \mathrm{eV}$. This can be explained on the basis of the optical conductivity presented in Fig. 4(b). When $\mu<$ $0.3 \mathrm{eV}$, the optical conductivity of phosphorene falls under an insulating regime yielding low coherence values since, in this case, only very weakly confined TE modes are supported. For $\mu>0.3 \mathrm{eV}$, we are in the metallic regime, and phosphorene plasmons are excited by the dipole, enhancing the decay rates and the anisotropy, yielding high coherence values as discussed earlier.

Recently, experiments have shown that $\alpha-\mathrm{MoO}_{3}$ exhibits in-plane hyperbolicity $[23,68]$ in the midinfrared range due to anisotropic phonon polaritons. Interestingly, BLG 
excitons can be tuned electrically in the hyperbolic regime of $\mathrm{MoO}_{3}$ [25]. This is our second proposed heterostructure for realizing tunable valley coherence in the $\alpha-\mathrm{MoO}_{3} / \mathrm{BLG}$ heterostructure as shown in Fig. 4(d). Here, electrical tunability can be achieved by tuning the frequency of the excitons of the BLG via the Stark effect. As per our general guidelines in Fig. 3, high valley coherence in the hyperbolic regime occurs in the large insulating and low metallic region which is near $800 \mathrm{~cm}^{-1}$. Additionally, we also see a large valley coherence at $550 \mathrm{~cm}^{-1}$ at the transition region between the elliptical and the hyperbolic band 1 region of $\mathrm{MoO}_{3}$. In Fig. 4(f), we show the $Q$ vs the exciton frequency for $40-\mathrm{nm} \mathrm{MoO}_{3}$ thicknesses. As shown in Ref. [23], the in-plane polariton wave vector decreases with increasing thickness. The thickness presents another knob for selecting the wave vector at a given frequency and spacer thickness-thus, controlling the spectral location of the valley coherence maxima. To achieve the exciton energies in BLG as given in Fig. 4(f), one needs to apply displacement fields ranging from 0.69 to $1.37 \mathrm{~V} / \mathrm{nm}$ which is well within the experimental reach [25].
To summarize, we discussed how the optical anisotropy of the polaritons supported by layered material, which is placed near a valleytronic 2D material results in enhanced spontaneous valley coherence in the heterostructure and proposes a way to measure the generated spontaneous valley coherence experimentally. We explored the phase space of the optical conductivity tensor of such an anisotropic 2D material and presented a $Q$ map in the hyperbolic and elliptical regions to guide experimental designs. As example systems, we showed how this valley coherence could be tuned electrostatically in the near-infrared using the phosphorene/TMDC heterostructure and in the midinfrared in the $\alpha-\mathrm{MoO}_{3} / \mathrm{BLG}$ heterostructure. Our proposed tunable valley coherence in 2D heterostructures offers a natural materials platform for quantum valley physics and applications [69].

A.K. acknowledges funding from the Department of Science and Technology Grants No. SB/S2/RJN-110/2017, No. ECR/2018/001485, and No. DST/NM/NS-2018/49. We thank K. Khaliji for helpful discussions.
[1] N. Yu and F. Capasso, Nature Mater. 13, 139 (2014).

[2] H. Chen, C. T. Chan, and P. Sheng, Nature Mater. 9, 387 (2010).

[3] S. Jahani and Z. Jacob, Nat. Nanotechnol. 11, 23 (2016).

[4] A. Poddubny, I. Iorsh, P. Belov, and Y. Kivshar, Nat. Photonics 7, 948 (2013).

[5] K. M. Schulz, H. Vu, S. Schwaiger, A. Rottler, T. Korn, D. Sonnenberg, T. Kipp, and S. Mendach, Phys. Rev. Lett. 117, 085503 (2016).

[6] L. Li, W. Wang, T. S. Luk, X. Yang, and J. Gao, ACS Photonics 4, 501 (2017).

[7] T. U. Tumkur, L. Gu, J. K. Kitur, E. E. Narimanov, and M. A. Noginov, Appl. Phys. Lett. 100, 161103 (2012).

[8] P. K. Jha, M. Mrejen, J. Kim, C. Wu, Y. Wang, Y. V. Rostovtsev, and X. Zhang, Phys. Rev. Lett. 116, 165502 (2016).

[9] D. Lu and Z. Liu, Nat. Commun. 3, 1205 (2012).

[10] P. A. Belov, Microwave Opt. Technol. Lett. 37, 259 (2003).

[11] X. Liu and Z. Zhang, ACS Photonics 2, 1320 (2015).

[12] Y. Guo and Z. Jacob, Opt. Express 21, 15014 (2013).

[13] Y. Guo, C. L. Cortes, S. Molesky, and Z. Jacob, Appl. Phys. Lett. 101, 131106 (2012).

[14] K. V. Sreekanth, Y. Alapan, M. ElKabbash, E. Ilker, M. Hinczewski, U. A. Gurkan, A. D. Luca, and G. Strangi, Nature Mater. 15, 621 (2016).

[15] Y. Guo, W. Newman, C. L. Cortes, and Z. Jacob, Adv. OptoElectron. 2012, 452502 (2012).

[16] D. R. Smith, D. C. Vier, T. Koschny, and C. M. Soukoulis, Phys. Rev. E 71, 036617 (2005).

[17] K. Korzeb, M. Gajc, and D. A. Pawlak, Opt. Express 23, 25406 (2015).

[18] H. Zhang, ACS Nano 9, 9451 (2015).

[19] T. Low, A. Chaves, J. D. Caldwell, A. Kumar, N. X. Fang, P. Avouris, T. F. Heinz, F. Guinea, L. Martin-Moreno, and F. Koppens, Nature Mater. 16, 182 (2016).

[20] M. Esslinger, R. Vogelgesang, N. Talebi, W. Khunsin, P. Gehring, S. de Zuani, B. Gompf, and K. Kern, ACS Photonics 1, 1285 (2014).
[21] E. van Veen, A. Nemilentsau, A. Kumar, R. Roldán, M. I. Katsnelson, T. Low, and S. Yuan, Phys. Rev. Appl. 12, 014011 (2019).

[22] C. Wang, G. W. Zhang, S. Y. Huang, Y. G. Xie, and H. Yan, Adv. Opt. Mater. 8, 1900996 (2020).

[23] W. Ma, P. Alonso-González, S. Li, A. Y. Nikitin, J. Yuan, J. Martín-Sánchez, J. Taboada-Gutiérrez, I. Amenabar, P. Li, S. Vélez, C. Tollan, Z. Dai, Y. Zhang, S. Sriram, K. KalantarZadeh, S.-T. Lee, R. Hillenbrand, and Q. Bao, Nature (London) 562, 557 (2018).

[24] G. Wang, A. Chernikov, M. M. Glazov, T. F. Heinz, X. Marie, T. Amand, and B. Urbaszek, Rev. Mod. Phys. 90, 021001 (2018).

[25] L. Ju, L. Wang, T. Cao, T. Taniguchi, K. Watanabe, S. G. Louie, F. Rana, J. Park, J. Hone, F. Wang, and P. L. McEuen, Science 358, 907 (2017).

[26] J. R. Schaibley, H. Yu, G. Clark, P. Rivera, J. S. Ross, K. L. Seyler, W. Yao, and X. Xu, Nat. Rev. Mater. 1, 16055 (2016).

[27] F. Bussolotti, H. Kawai, Z. E. Ooi, V. Chellappan, D. Thian, A. L. C. Pang, and K. E. J. Goh, Nano Futures 2, 032001 (2018).

[28] S. A. Vitale, D. Nezich, J. O. Varghese, P. Kim, N. Gedik, P. Jarillo-Herrero, D. Xiao, and M. Rothschild, Small 14, 1801483 (2018).

[29] A. M. Jones, H. Yu, N. J. Ghimire, S. Wu, G. Aivazian, J. S. Ross, B. Zhao, J. Yan, D. G. Mandrus, D. Xiao, W. Yao, and X. $\mathrm{Xu}$, Nat. Nanotechnol. 8, 634 (2013).

[30] P. K. Jha, N. Shitrit, X. Ren, Y. Wang, and X. Zhang, Phys. Rev. Lett. 121, 116102 (2018).

[31] G. S. Agarwal, Phys. Rev. Lett. 84, 5500 (2000).

[32] D. Kornovan, M. Petrov, and I. Iorsh, Phys. Rev. A 100, 033840 (2019).

[33] P. K. Jha, X. Ni, C. Wu, Y. Wang, and X. Zhang, Phys. Rev. Lett. 115, 025501 (2015).

[34] L. Qiu, C. Chakraborty, S. Dhara, and A. N. Vamivakas, Nat. Commun. 10, 1513 (2019).

[35] D. Dzsotjan, J. Kästel, and M. Fleischhauer, Phys. Rev. B 84, 075419 (2011). 
[36] S. Hughes and G. S. Agarwal, Phys. Rev. Lett. 118, 063601 (2017).

[37] See Supplemental Material at http://link.aps.org/supplemental/ 10.1103/PhysRevB.102.045416 for the decay rate calculations and rate equations of the excitonic population .

[38] K. Hao, G. Moody, F. Wu, C. K. Dass, L. Xu, C.-H. Chen, L. Sun, M.-Y. Li, L.-J. Li, A. H. MacDonald, and X. Li, Nat. Phys. 12, 677 (2016).

[39] H. Zeng, J. Dai, W. Yao, D. Xiao, and X. Cui, Nat. Nanotechnol. 7, 490 (2012).

[40] G. Kioseoglou, A. T. Hanbicki, M. Currie, A. L. Friedman, D. Gunlycke, and B. T. Jonker, Appl. Phys. Lett. 101, 221907 (2012).

[41] B. R. Carvalho, Y. Wang, S. Mignuzzi, D. Roy, M. Terrones, C. Fantini, V. H. Crespi, L. M. Malard, and M. A. Pimenta, Nat. Commun. 8, 14670 (2017).

[42] M. Z. Maialle, E. A. de Andrada e Silva, and L. J. Sham, Phys. Rev. B 47, 15776 (1993).

[43] M. M. Glazov, T. Amand, X. Marie, D. Lagarde, L. Bouet, and B. Urbaszek, Phys. Rev. B 89, 201302(R) (2014).

[44] C. R. Zhu, K. Zhang, M. Glazov, B. Urbaszek, T. Amand, Z. W. Ji, B. L. Liu, and X. Marie, Phys. Rev. B 90, 161302(R) (2014).

[45] E. M. Purcell, Phys. Rev. 69, 674 (1946).

[46] L. Novotny and B. Hecht, Principles of Nano-Optics (Cambridge University Press, Cambridge, UK, 2006).

[47] A. Lakhtakia, Int. J. Infrared Millimeter Waves 13, 161 (1992).

[48] J. S. Gomez-Diaz, M. Tymchenko, and A. Alù, Opt. Mater. Express 5, 2313 (2015).

[49] G. Széchenyi, M. Vigh, A. Kormányos, and J. Cserti, J. Phys.: Condens. Matter 28, 375802 (2016).

[50] J. S. Gomez-Diaz, M. Tymchenko, and A. Alù, Phys. Rev. Lett. 114, 233901 (2015).

[51] S. A. Mikhailov and K. Ziegler, Phys. Rev. Lett. 99, 016803 (2007).

[52] V. D. Karanikolas, C. A. Marocico, P. R. Eastham, and A. L. Bradley, Phys. Rev. B 94, 195418 (2016).
[53] A. Nemilentsau, T. Low, and G. Hanson, Phys. Rev. Lett. 116, 066804 (2016).

[54] A. Kumar, T. Low, K. H. Fung, P. Avouris, and N. X. Fang, Nano Lett. 15, 3172 (2015).

[55] F. Xia, H. Wang, J. C. M. Hwang, A. H. Castro Neto, and L. Yang, Nat. Rev. Phys. 1, 306 (2019).

[56] A. Carvalho, M. Wang, X. Zhu, A. S. Rodin, H. Su, and A. H. Castro Neto, Nat. Rev. Mater. 1, 16061 (2016).

[57] G. C. Constantinescu and N. D. M. Hine, Nano Lett. 16, 2586 (2016).

[58] Y. Tatsumi, K. Ghalamkari, and R. Saito, Phys. Rev. B 94, 235408 (2016).

[59] C. Ruppert, O. B. Aslan, and T. F. Heinz, Nano Lett 14, 6231 (2014).

[60] T. Low, A. S. Rodin, A. Carvalho, Y. Jiang, H. Wang, F. Xia, and A. H. Castro Neto, Phys. Rev. B 90, 075434 (2014).

[61] X. Li, Z. Yu, X. Xiong, T. Li, T. Gao, R. Wang, R. Huang, and Y. Wu, Sci. Adv. 5, eaau3194 (2019).

[62] M. Buscema, D. J. Groenendijk, G. A. Steele, H. S. J. van der Zant, and A. Castellanos-Gomez, Nat. Commun. 5, 4651 (2014).

[63] N. Youngblood, C. Chen, S. J. Koester, and M. Li, Nat. Photonics 9, 247 (2015).

[64] S. P. Koenig, R. A. Doganov, L. Seixas, A. Carvalho, J. Y. Tan, K. Watanabe, T. Taniguchi, N. Yakovlev, A. H. Castro Neto, and B. Özyilmaz, Nano Lett. 16, 2145 (2016).

[65] D. Xiang, C. Han, J. Wu, S. Zhong, Y. Liu, J. Lin, X.-A. Zhang, W. P. Hu, B. Özyilmaz, A. H. Castro Neto, A. T. S. Wee, and W. Chen, Nat. Commun. 6, 6485 (2015).

[66] R. Petersen, T. G. Pedersen, and F. Javier García de Abajo, Phys. Rev. B 96, 205430 (2017).

[67] Y. Saito and Y. Iwasa, ACS Nano 9, 3192 (2015).

[68] Z. Zheng, N. Xu, S. L. Oscurato, M. Tamagnone, F. Sun, Y. Jiang, Y. Ke, J. Chen, W. Huang, W. L. Wilson, A. Ambrosio, S. Deng, and H. Chen, Sci. Adv. 5, eaav8690 (2019).

[69] Y. S. Ang, S. A. Yang, C. Zhang, Z. Ma, and L. K. Ang, Phys. Rev. B 96, 245410 (2017). 